\title{
Flutuação populacional de moscas-das-frutas (Diptera, Tephritidae) em pomares de pessegueiro e maracujazeiro em Iraceminha, Santa Catarina
}

\author{
Sheila Alberti ${ }^{1}$ \\ Gilson Marcos Bogus ${ }^{1}$ \\ Flávio Roberto Mello Garcia $2 *$ \\ ${ }^{1}$ Universidade Comunitária Regional de Chapecó \\ PPG em Ciências Ambientais, Laboratório de Entomologia, Chapecó - SC, Brasil \\ ${ }^{2}$ Universidade Federal de Pelotas, Instituto de Biologia \\ Departamento de Zoologia e Genética, Laboratório de Ecologia de Insetos \\ Caixa Postal 35, CEP 96010-900, Pelotas - RS, Brasil \\ * Autor para correspondência \\ flavio.garcia@pq.cnpq.br
}

Submetido em 15/06/2011

Aceito para publicação em 19/12/2011

\section{Resumo}

O objetivo do estudo foi conhecer a flutuação populacional das moscas-das-frutas em pomares de pessegueiro e maracujazeiro no município de Iraceminha, Santa Catarina. Para o levantamento foram instalados frascos “caça-moscas" do tipo McPhail, contendo glicose invertida a 10\%, no período de abril de 2006 a março de 2007. As moscas capturadas foram identificadas no Laboratório de Entomologia da UNOCHAPECÓ. As fêmeas coletadas pertencem a 12 espécies e quatro gêneros. Os adultos de Anastrepha fraterculus (Wiedeman, 1830) ocorreram em todas as estações do ano, com pico populacional em outubro/2006 nos pomares de pessegueiro. Os níveis populacionais de Anastrepha grandis (Macquat, 1846) mantiveram-se mais elevados durante os primeiros cinco meses da pesquisa, no pomar de maracujazeiro. Foi constatada baixa infestação por moscas dos gêneros Ceratitis, Blepharoneura e Tomoplagia, em relação às moscas do gênero Anastrepha. Os resultados mostraram que a presença das moscas nos pomares está associada à disponibilidade de alimento e não com as condições climáticas.

Palavras-chave: Anastrepha; Insecta; Nível populacional; Pomares

\section{Abstract}

Population fluctuation of fruit flies (Diptera, Tephritidae) in peach and passion fruit orchards in Iraceminha, Santa Catarina. The objective of this study was learn more about the population fluctuation of fruit flies in peach and passion fruit orchards in the municipality of Iraceminha, Santa Catarina. In order to carry out the survey, McPhail traps were set up with 10\% inverted glucose from April 2006 to March 2007. Captured flies were identified at the Laboratório de Entomologia at UNOCHAPECÓ. The collected females belonged to twelve species and four genera. The adults of Anastrepha fraterculus were trapped during all seasons, with a population peak in October 2006 in the peach orchards. The population levels of Anastrepha grandis remained higher during the first five months in the passion fruit orchard. There were low infestation rates by flies of the 
genera Ceratitis, Blepharoneura and Tomoplagia when compared to flies of the genus Anastrepha. The results showed that the presence of flies in the orchards was associated with the availability of host fruit and not with climatic conditions.

Key words: Anastrepha; Insecta; Orchards; Population level

\section{Introdução}

O estado de Santa Catarina sobressai-se no cenário nacional como um grande produtor de frutas, principalmente de clima temperado (NORA et al., 2000) e os produtores preocupam-se com a qualidade dos frutos produzidos, principalmente devido aos danos que podem ser ocasionados por moscas-das-frutas.

As moscas-das-frutas da família Tephritidae principalmente aquelas do gênero Anastrepha e Ceratitis capitata (Wied., 1830), constituem um dos principais problemas fitossanitários da fruticultura brasileira (GARCIA., 2009).

Em estudos de flutuação populacional de moscasdas-frutas em pessegueiro conduzidos em quatro municípios da região oeste de Santa Catarina, constatouse uma maior incidência de Anastrepha fraterculus (Wied., 1830) nos meses de dezembro e janeiro, concomitante com a frutificação do pessegueiro (GARCIA et al., 2003). A dinâmica populacional das moscas-das-frutas sofre interferências diretas da disponibilidade de hospedeiros e das variações dos fatores climáticos (GARCIA, 2009).

Portanto, o objetivo deste trabalho foi verificar a flutuação populacional de moscas-das-frutas em pomares de maracujazeiro e pessegueiro no município de Iraceminha, Oeste de Santa Catarina, e correlacionar os níveis populacionais dos tefritídeos com os ciclos das culturas e os fatores climáticos.

\section{Materiais e Métodos}

\section{Coletas}

O experimento foi conduzido em pomares de maracujazeiro e pessegueiro ("A" e "B"), situados no município de Iraceminha, Santa Catarina (26²9'S, $53^{\circ} 16^{\prime} \mathrm{W}$ e altitude aproximada de $450 \mathrm{~m}$ ), no período de abril de 2006 a março de 2007. O clima predominante nesta região é o mesotérmico-úmido, com verão quente (PREFEITURA MUNICIPAL DE IRACEMINHA, 2007),

$\mathrm{Na}$ coleta das moscas foram utilizadas 20 armadilhas caça-moscas do tipo McPhail, contendo $200 \mathrm{~mL}$ de solução aquosa de glicose invertida a 10\% (GARCIA et al., 1999), as quais estavam instaladas a uma altura aproximada de $1,5 \mathrm{~m}$ na copa das plantas.

Foram realizadas coletas semanais, para a troca da solução atrativa e coleta dos tefritídeos. Os insetos foram acondicionados em frascos etiquetados, contendo álcool $70 \%$, posteriormente foram sexados, contados e levados para o Laboratório de Entomologia da UNOCHAPECÓ para identificação.

\section{Identificação}

As identificações das espécies foram baseadas nos caracteres morfológicos das fêmeas, usando o padrão alar, padrão torácico, mediotergito, subescutelo e exame ventral do acúleo. As moscas foram colocadas ventralmente em uma lâmina sob a lupa, e com auxilio de dois estiletes, foi extrovertido o acúleo. Em seguida, a lâmina foi colocada sob o microscópio estereoscópico e sobre esta se colocou uma gota de glicerina para o exame sob aumento de 40 ou 100 vezes.

A identificação das espécies de Anastrepha foi baseada em Steyskal (1977) e Zucchi (2000), e os outros gêneros foram identificados através das chaves confeccionadas por Foote (1980).

\section{Análises}

Foi realizado estudo de flutuação populacional apenas das espécies de moscas-das-frutas que apresentaram maiores índices de abundância em trabalho realizado publicado anteriormente nos mesmos pomares (ALBERTI et al., 2009). 
Foram feitas anotações sobre a fenologia do pessegueiro e maracujazeiro Estas informações serviram para comparar com as etapas da cultura e a flutuação populacional dos tefritídeos.

Os dados climáticos do município de Iraceminha, representados por temperatura máxima, temperatura média, temperatura mínima e umidade relativa do ar, para cada semana de coleta, foram calculados a partir dos dados da Estação Experimental da EPAGRI/CIRAM.

\section{Resultados e Discussão}

Foram capturados 697 exemplares de moscas-dasfrutas de 12 espécies pertencentes a quatro gêneros: Anastrepha barbiellinii Lima, 1938, Anastrepha dissimilis Stone, 1942, Anastrepha fraterculus, Anastrepha grandis Macquart, 1846, Anastrepha montei Lima, 1934, Anastrepha obliqua (Macquart, 1835), Anastrepha pseudoparallela (Loew, 1873), Anastrepha sororcula Zucchi, 1979, Anastrepha xanthochaeta Hendel, 1914, Blepharoneura sp., C. capitata e Tomoplagia sp.

Os níveis populacionais de $A$. grandis mantiveramse mais elevados durante os primeiros cinco meses do estudo, sendo que seu pico populacional ocorreu no mês de junho de 2006 (Figura 1). Anastrepha fraterculus apresentou um pico em março de 2007. A elevada dominância de apenas uma ou duas espécies de moscasdas-frutas também foi verificada por diversos outros pesquisadores, em trabalhos similares realizados no Brasil (GARCIA; CORSEUIL, 1998; URAMOTO et al., 2003; 2004; ARAÚJO et al., 2005). Já, A. barbiellinii apresentou nível populacional mais elevado durante os meses de outubro de 2006 e março de 2007.

No pessegueiro "A", A. fraterculus esteve presente na maior parte do período de amostragem, exceto nos meses de julho/2006 e março/2007 (Figura 2). Seu pico populacional foi em outubro de 2006, coincidindo com a época de maturação dos frutos. Segundo Nora et al. (2000), a grande diversidade de plantas hospedeiras nativas e cultivadas em Santa Catarina, com diferentes épocas de frutificação, facilitam a reprodução sucessiva de $A$. fraterculus durante o ano todo.

É interessante ressaltar que $10 \%$ das moscas da espécie $A$. fraterculus apresentavam má formação em dentes do acúleo do ovipositor, isso pode levantar a hipótese de ser uma característica morfológica desta espécie no Oeste Catarinense, pois esta anomalia já havia sido constatada em outros espécimes oriundos desta mesma região (ZUCCHI, 2001).

FIGURA 1: Flutuação populacional de fêmeas de Anastrepha obtidas do pomar de maracujazeiro, no município de Iraceminha, SC.

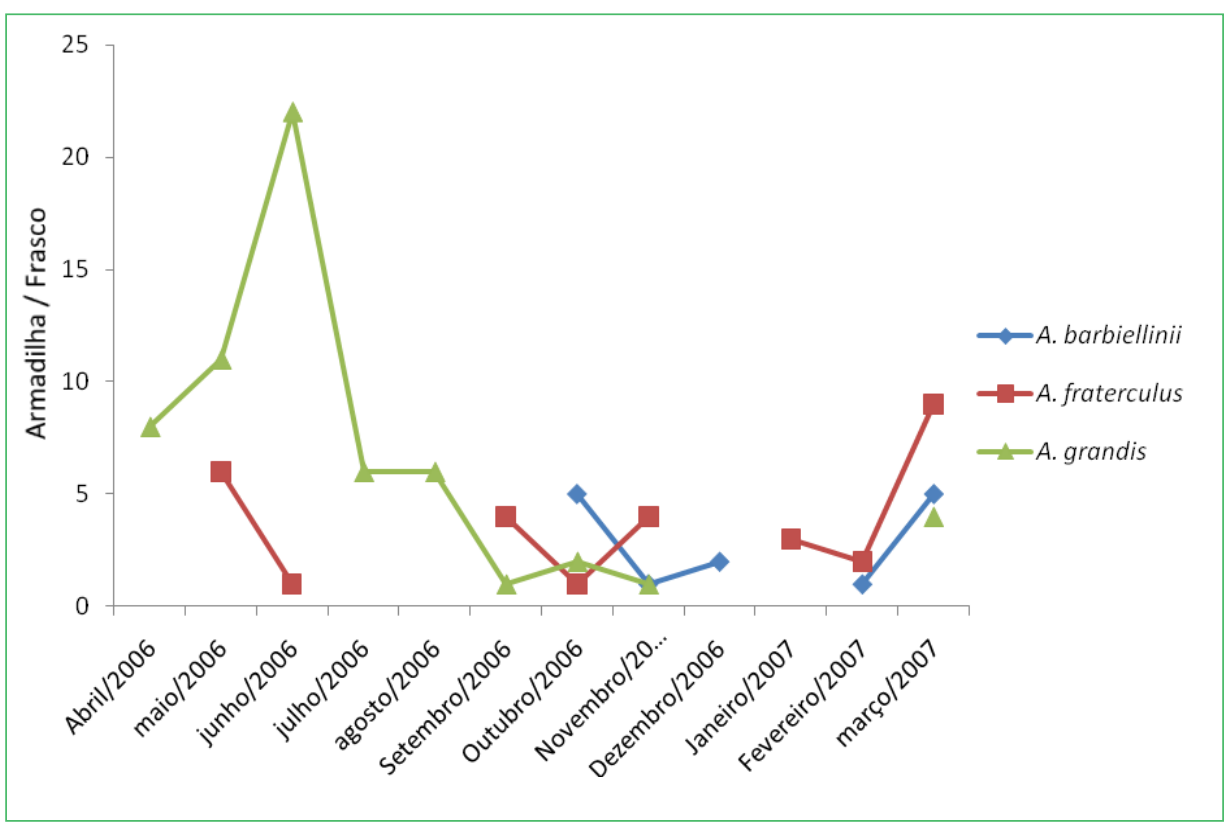


FIGURA 2: Flutuação populacional de A. fraterculus e A. grandis obtidas do pomar de pessegueiro “A”, no município de Iraceminha, SC.

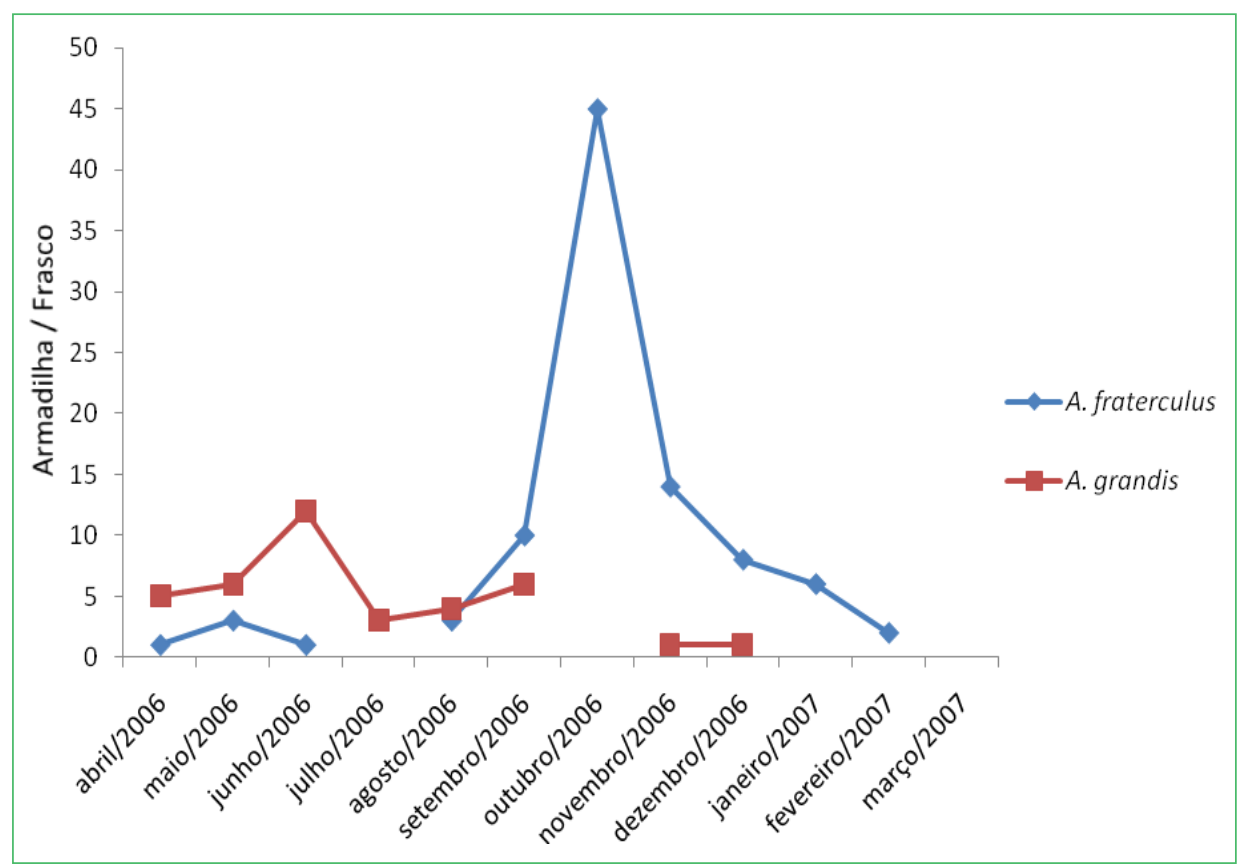

No pomar de pessegueiro "B", ao longo dos meses de setembro e outubro de 2006, foi coletado cerca de $68 \%$ de todos os indivíduos da espécie $A$. fraterculus capturados durante o período de realização do presente estudo (Figura 3).

Verificou-se a presença de $A$. grandis nos sete primeiros meses e no último mês do estudo. Em geral, os picos populacionais desses tefritídeos no pomar de pessegueiro " $\mathrm{B}$ " ocorreram durante maio e junho de 2006, período em que não há frutos no pomar, tal fato pode ser atribuído a ocorrência de cucurbitáceas nas proximidades do pomar, posto que $A$. grandis possui apenas hospedeiros dessa família (GARCIA; NORRBOM, 2011).

FIGURA 3: Flutuação populacional do gênero Anastrepha obtidas do pomar de pessegueiro "B", no município de Iraceminha, Oeste Catarinense, no período de abril de 2006 a março de 2007.

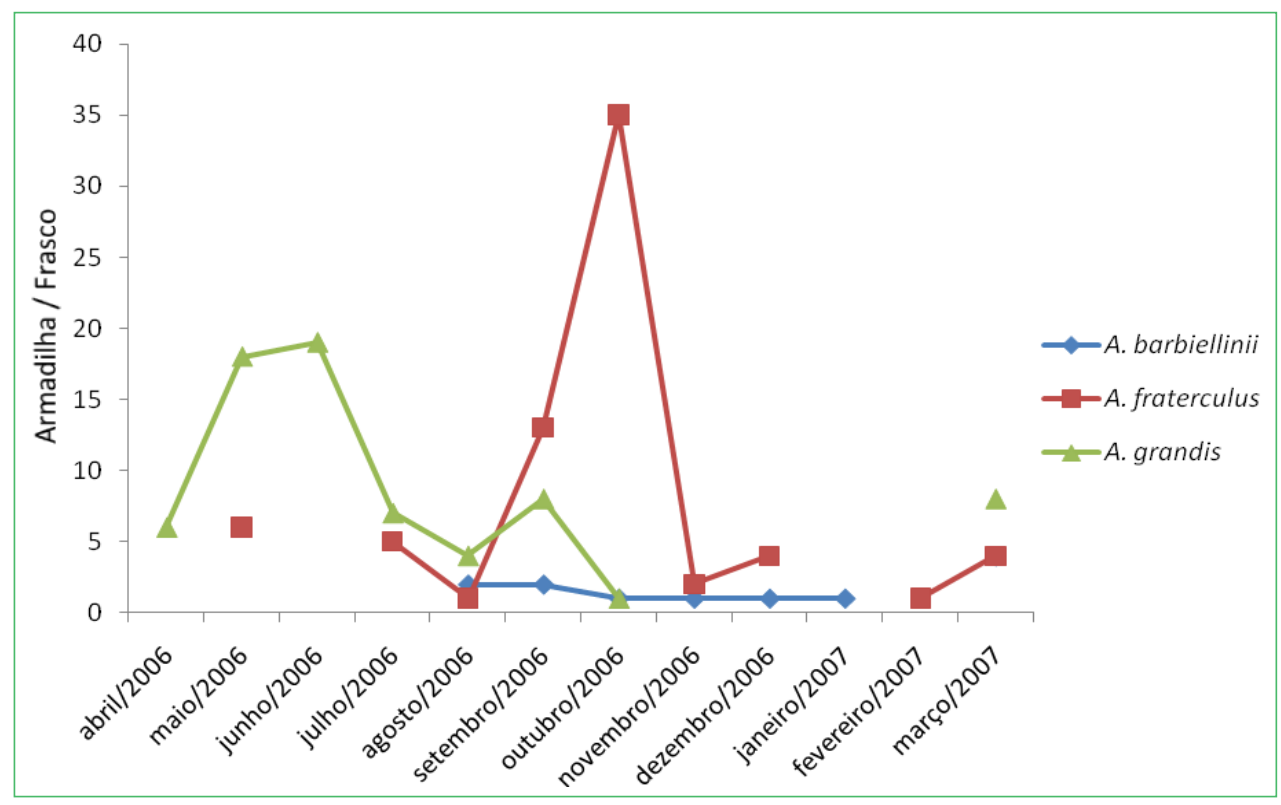


Para C. capitata, o pico populacional ocorreu em dezembro/2006, período que compreende a época de frutificação do pessegueiro, um dos principais hospedeiros da mosca-do-mediterrâneo.

As espécies $A$. barbiellinii, A. dissimilis, $A$. pseudoparallela, A. sororcula e Blepharoneura sp. apresentaram um baixo pico populacional. Estudos desenvolvidos por Aluja et al. (1996), porém, sugerem que esse tipo de comportamento seja considerado normal, visto que embora diversas espécies de tefritídeos estejam presentes em um pomar, apenas uma ou duas espécies representam mais de $90 \%$ de todas as moscas coletadas em armadilhas.

Através da análise de regressão múltipla entre o número de moscas coletadas por semana de $A$. fraterculus e $A$. grandis, não se obteve diferenças estatísticas significativas entre os fatores climáticos e os níveis populacionais dessas espécies, esse mesmo resultado foi obtido por Garcia e Lara (2006), todavia difere dos resultados obtidos por Garcia e Corseuil (1998; 1999), Garcia et al. (2003); Uramoto et al. (2004), Garcia e Lara (2006) e Zilli e Garcia (2010). Isto deve-se, possivelmente, ao baixo número de espécimes coletados.

A partir dos resultados obtidos pode-se concluir que:

- O pico populacional de $A$. fraterculus coincide com a época de maturação do pêssego.

- Não se obteve correlação significativa entre os fatores climáticos e os níveis populacionais de A. fraterculus e $A$. grandis.

\section{Referências}

ALBERTI, S.; GARCIA, F. R. M.; BOGUS, G. M. Moscasdas-frutas (Diptera: Tephritidae) em pomares de pessegueiro e maracujazeiro no município de em Iraceminha, Santa Catarina. Ciência Rural, Santa Maria, v. 39, p. 1565-1568, 2009.

ALUJA, M.; CELEDONIO-HURTADO, H.; LIEDO, P.; CABRERA, M.; CASTILLO, F.; GUILLÉN, J.; RIOS, E. Seasonal population fluctuations and ecological implications for management of Anastrepha fruit flies (Diptera: Tephritidae) in commercial orchards in Southern Mexico. Journal of Economic Entomology, Riverside, v. 89, n. 3, p. 654-667, 1996.

ARAÚJO, E. L.; MEDEIROS, M. K. M.; SILVA, V. E.; ZUCCHI, R. A. Moscas-das-frutas (Diptera: Tephritidae) no semi-árido do Rio Grande do Norte: plantas hospedeiras e índices de infestação. Neotropical Entomology, Piracicaba, v. 34, n. 6, p. 889-894, 2005.
FOOTE, R. H. Fruit fly genera south of the United States (Diptera: Tephritidae). Washington D. C.: Science Education Administration, $1980.79 \mathrm{p}$.

GARCIA, F. R. M. Fruit fly: biological and ecological aspects. In: BANDEIRA, R. R. (Ed.). Current trends in fruit flies control on perennial crops and research prospects. Kerala: Transworld Research Network, 2009. p. 1-35.

GARCIA, F. R. M.; CAMPOS, J. V.; CORSEUIL, E. Avaliação de atrativos na captura de adultos de Anastrepha fraterculus (Wied., 1830) (Diptera: Tephritidae). Biociências, Porto Alegre, v. 7, n. 1, p. 43-50, 1999 .

GARCIA, F. R. M; CAMPOS, J. V.; CORSEUIL, E. Flutuação populacional de Anastrepha fraterculus (Wiedemann, 1830) (Diptera, Tephritidae) na Região Oeste de Santa Catarina, Brasil. Revista Brasileira de Entomologia, Curitiba, v. 47, n. 3, p. 415420, 2003.

GARCIA, F. R. M.; CORSEUIL, E. Flutuação populacional de Anastrepha fraterculus (Wiedemann) e Ceratitis capitata (Wiedemann) (Diptera: Tephritidae) em pomares de pessegueiro em Porto Alegre, Rio Grande do Sul. Revista Brasileira de Zoologia, Curitiba, v. 15, n. 1, p. 153-158, 1998.

GARCIA, F. R. M.; CORSEUIL, E. Influência de fatores climáticos sobre moscas-das-frutas (Diptera: Tephritidae) em pomares de pessegueiro em Porto Alegre, Rio Grande do Sul. Revista da Faculdade de Zootecnia, Veterinária e Agronomia, Uruguaiana, v. 5/6, n. 1, p. 71-75, 1999.

GARCIA, F. R. M.; LARA, D. B. de. Análise faunística e flutuação populacional de moscas-das-frutas (Diptera, Tephritidae) em pomar cítrico no município de Dionísio Cerqueira, Santa Catarina. Biotemas, Florianópolis, v. 19, n. 3, p. 65-70, 2006.

GARCIA, F. R. M.; NORRBOM, A. L. Tephritoid flies (Diptera, Tephritoidea) and their plant hosts from the state of Santa Catarina in southern Brazil. Florida Entomologist, Gainesville, v. 94, p. 151-157, 2011

NORA, I.; HICKEL, E. R.; PRANDO, H. F. Moscas-das-frutas nos estados brasileiros: Santa Catarina. In: MALAVASI, A.; ZUCCHI, R. A. (Ed.). Moscas-das-frutas de importância econômica no Brasil: conhecimento básico e aplicado. Ribeirão Preto: Holos Editora, 2000. p. 271-275.

PREFEITURA MUNICIPAL DE IRACEMINHA. Aspectos geográficos do município. Iraceminha: município integrado à rede catarinense de informações municipais RedeCIM, 2007. Disponível em <http://www.iraceminha.sc.gov.br/conteudo/?item $=15369 \& \mathrm{fa}=4703 \&$ PHPSESSID $=53 \mathrm{beb} 2 \mathrm{a} 5027 \mathrm{~d} 6 \mathrm{~b} 24 \mathrm{~d} 136 \mathrm{a} 1 \mathrm{e} 744$ da6cb4>. Acesso em: 28 dez. 2007.

STEYSKAL, G. C. Pictorial key to species of the genus Anastrepha (Diptera: Tephritidae). Washington: The Entomological Society of Washington, 1977. $35 \mathrm{p}$.

URAMOTO, K.; WALDER, J. M. M.; ZUCCHI, R. A. Flutuação populacional de moscas-das-frutas do gênero Anastrepha Schiner, 1868 (Diptera, Tephritidae) no Campus "Luiz de Queiroz", Piracicaba, São Paulo. Arquivos do Instituto Biológico de São Paulo, São Paulo, v. 70, n. 4, p. 459-465, 2003.

URAMOTO, K.; WALDER, J. M. M.; ZUCCHI, R. A. Biodiversidade de moscas-das-frutas do gênero Anastrepha (Diptera, Tephritidae) no campus da ESALQ-USP, Piracicaba, São Paulo. Revista Brasileira de Entomologia, Curitiba, v. 48, n. 3, p. 409-414, 2004. 
ZILLI, G. N.; GARCIA, F. R. M. Análise faunística e flutuação populacional de moscas-das-frutas (Diptera, Tephritidae) em pomar de Citrus sinensis no município de Chapecó, Santa Catarina. Biodiversidade Pampeana, Uruguaiana, v. 8, p. 39-45, 2010.

ZUCCHI, R. A. Taxonomia. In: MALAVASI, A.; ZUCCHI, R. A. (Eds). Moscas-das-frutas de importância econômica no Brasil: conhecimento básico e aplicado. Ribeirão Preto: Holos Editora, 2000. p.13-24.

ZUCCHI, R. A. Mosca-do-Mediterrâneo, Ceratitis capitata (Diptera, Tephritidae). In: VILELA, E. F.; ZUCCHI, R. A.; CANTOR, F. (Eds). Histórico e impacto de pragas introduzidas no Brasil. Ribeirão Preto: Holos Editora, 2001. p. 15-22. 